\title{
IMPROVEMENT IN RABBIT CORNEAL CELL SUSPENSION VIABILITY AFTER FREEZING WITH GINGKO BILOBA EXTRACT
}

\author{
Murad Aktan ${ }^{1}$, Berrin Okka ${ }^{2}$, Mehmet Okka ${ }^{2}$, Selcuk Duman ${ }^{1}$
}

Selcuk University, Meram Medical Faculty, Konya, Turkey: Histology-Embryology Deptartment ${ }^{1}$; University of Wisconsin, Medical School, Madison, WI USA, Ophthalmology Department ${ }^{2}$

Summary: We investigated whether the addition of Gingko Biloba extract (EGb 761) to rabbit corneal epithelial medium before cell freezing improved cell viability after freezing then thawing. After removal of corneas, they were treated with enzymes and the corneal epithelium was prepared as a single cell suspension in freezing media with or without EGb 761 . After freezing for two weeks then thawing, a higher cell viability was found in the cornea cell suspensions which had been frozen pretreated with EGb 761 in the media. The improvement with corneal cell viability with EGb 761 pretreatment is postulated to be based on the antioxidant capacity of the plant extract.

Key words: Cornea Epithelium; Gingko Biloba Extract; Cryopreservation

\section{Introduction}

Gingko Biloba tree leaf extract (EGb 761) is a plant product with many health benefits that have been documents for over 800 years. The antioxidative and scavenging effects of EGb 761 are thought to be beneficial for enhancing cognitive abilities in elderly people, protecting cells under UV oxidative stress, anti-clastogenic effects, enhancing nerve conduction velocity, erythrocyte membrane flexibility and many others as well (5).

During cell preservation by freezing, a cryoprotective agent is added to the freezing medium. In our study we aimed to evaluate an agent added to the medium before freezing, and which was removed from the medium during freezing process, and whether EGb 761 could provide additional protective effects that would result in enhanced cell viability upon thawing.

\section{Material and methods}

\section{Animals}

Male New Zealand white rabbits $(n=18)$ weighting 2.300-2760 gr with no prior medical or surgical experimental history were selected. Rabbits received tap water and standard rabbit chow ad libitum; housing in propylene cages $(55 \times 60 \times 35 \mathrm{~cm})$ was done in a 12 -hour light/dark cycle with, $20-24^{\circ} \mathrm{C}$ temperatures and $60 \pm 5$ humidity. No more than 3 rabbits were placed in a cage. All procedures were in accordance with the Council Directive of the European
Communities on the Protection of Animals Used for Experimental and Other Scientific Purposes (L358/1, November 24, 1986).

\section{Cornea Removal}

Rabbits were anesthetized i.m. ketamine hydrochloride (Ketalar; $25 \mathrm{mg} / \mathrm{kg}$ ) and xylazine hydrochloride (Rhompun; $5.0 \mathrm{mg} / \mathrm{kg}$ ). The eyes were anesthetized with 3-5 drops of oxybuprocaine. Corneas were removed full thickness by a $360^{\circ}$ limbal incision using micro scissors. After cornea removal, the rabbits were sacrificed with additional i.m. xylazine hydrochloride $(25.0 \mathrm{mg} / \mathrm{kg})$.

\section{Corneal Epithelial Cell Isolation}

The isolated corneas were first washed gently for 5-7 minutes in several exchanges of fresh phosphate buffered saline (PBS) supplemented with Penicillin G and Streptomycin sulfate (Irvine Scientific, Cat.No: 9316). They were then immediately transferred to a cell culture laboratory. Here each cornea (18 pairs, totally 36 cornea) was incubated with $15 \mathrm{ml}, 10 \%$ Tripsin-EDTA (Sigma T-4174) supplemented DMEM (Sigma, D6424) in $60 \mathrm{~mm}$ culture dishes (Nunclon, 150288). During this stage the cornea epithelium partially detached from the underlying stromal layer. Next the tissue pieces were placed in $10 \mathrm{ml} 15 \%$ FCS (Sigma F4135) supplemented DMEM (high FCS will help to stop the enzymatic reaction caused by Trypsin). Mechanical pulling with fine pens was done on partially detached epithelium under a stereomicroscope in order to 
separate the epithelium completely from the remaining cornea tissue. Large epithelial sheets were collected in medium and centrifuged at $1200 \mathrm{rpm}$ for 5 minutes. The epithelial tissue pellet was resuspended and placed in dishes containing 7-8 $\mathrm{ml}$ of DMEM (7.0 \% FCS supplemented) where it was finely minced with two surgical blades. Next, all tissue parts were collected in a bottle (Nunclone $100 \mathrm{ml}$ Polystyrene bottle for Seromed Ham's F12) containing 20 $\mathrm{ml}$ of DMEM supplemented with $10 \%$ Trypsin-EDTA and placed on a magnetic stirrer at $37^{\circ} \mathrm{C}$ for 25 minutes. Cells were harvested by collecting the upper medium (supernatant) following centrifugation. Fresh DMEM (20 ml) supplemented with $10 \%$ Trypsin-EDTA was added to the pellet remaining in the bottle and again stirred for another 25 minutes, centrifuged and supernatant harvested as done previously. All incubated pellets were pooled in one tube to estimate total cell number.

The total cell count was estimated after homogenizing the cell by pipetting then placing 10 microliters of the suspension on a Makler (SEFI-Medical, Israel) cell chamber and counting at $20 \mathrm{x}$ under a light microscope. The average from six counts was used to determine the total cell counts,

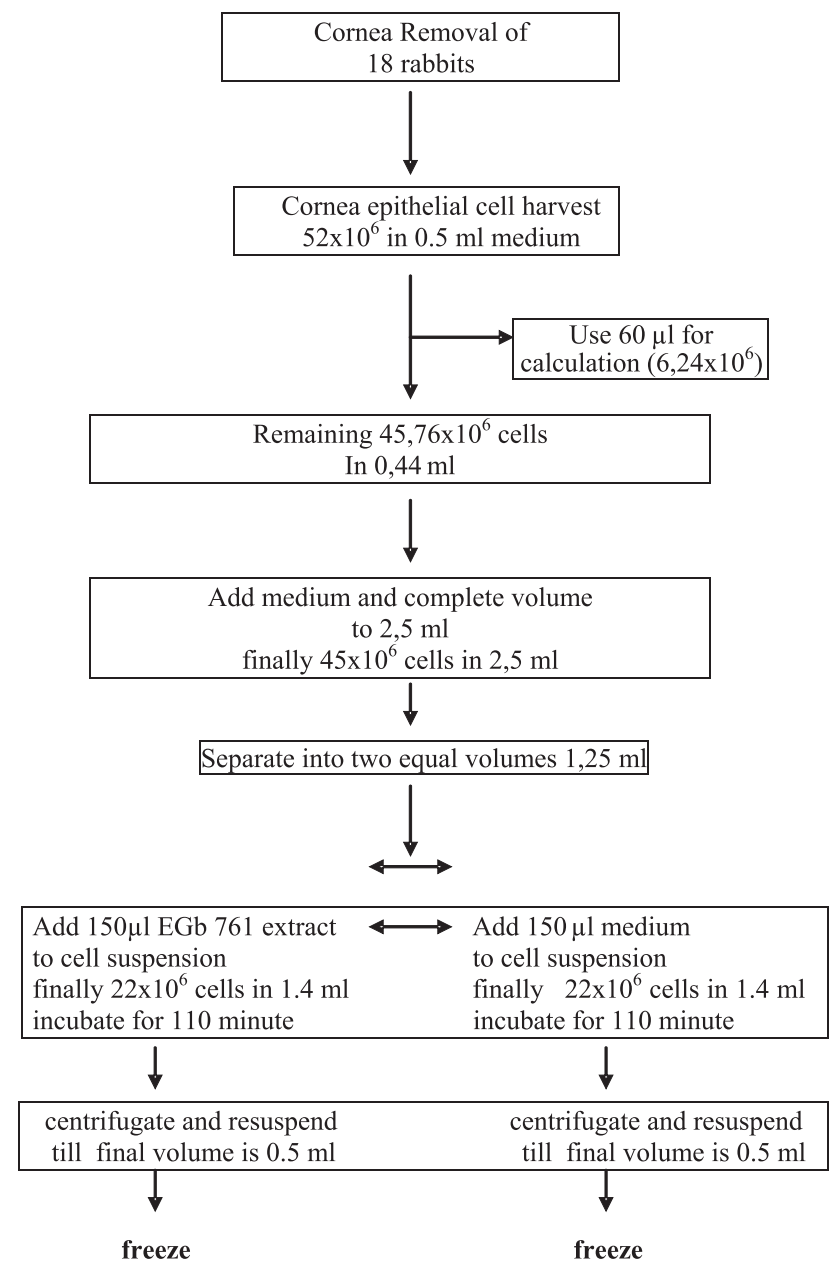

Fig. 1: Cell processing steps. which were 52x106 epithelial cells. This was divided equally into two tubes.

EGb 761 stock solution was adjusted to a final concentration of $40 \mathrm{mg} / \mathrm{ml}$. This solution was filtered through a 0.45-micron filter (Cameo 25ES, nitrocellulose). 150 microliters of the filtered solution EGb 761 was added to one tube. The volume of both tubes was adjusted so that each contained $1.4 \mathrm{ml}$ suspension containing approximately 20x106 epithelial cells. The suspensions were incubated $\left(37^{\circ} \mathrm{C}, 99 \%\right.$ humidity and $\left.6.0 \% \mathrm{CO}_{2}\right)$ for 110 minutes. Epithelial cells with no EGb 761 pretreatment are referred to as Group 1 (G1); EGb 761 pretreated cells are referred to as Group $2(\mathrm{G} 2)$. After the incubation period, $5.0 \mathrm{ml}$ of DMEM was added to each tube. The tubes were then centrifuged ( $240 \mathrm{~g}, 9$ minutes) to remove EGb 761 from the medium.

To estimate viability before freezing, Eosin Y (Merck 15936) testing was performed. Six separate samples from each group were taken and 200 cells were evaluated (Fig. 1).

\section{Freezing and Thawing}

Cryoprotectant Test Yolk Buffer (TYB) (Irvine Sci., Cat No: 9971) was added to the tubes slowly with the help of pipette in 5-6 minutes (100 microliter for each tube). Finally the cell suspension with cryoprotectant medium volume was 200 microliter and this was loaded to prelabeled straws by applying negative pressure at the filtered end attached to an insulin injector body. The free end of straw was sealed with a pens tip heated on fire. These straws were placed over liquid nitrogen (LN) steam for 20 minutes and then directly plunged into LN. The cells were stored in the LN tank for two weeks.

Thawing was done by simply removing the straws from the tank and leaving them at room temperature inside the laminar airflow cabinet for 6-7 minutes. After centrifugation (240g, 9 minutes) $1.0 \mathrm{ml}$ of DMEM was added to (resuspend with a pipette) the final pellet, which was then incubated $\left(37.0^{\circ} \mathrm{C}, 99 \%\right.$ humidity and $\left.6.0 \% \mathrm{CO}_{2}\right)$. A viability test was done as previously described at 0,1 and 2 hours after resuspension.

\section{Antioxidant Capacity}

A modification of Rice et al. method was used to determine the antioxidant capacity of DMEM, EGb 761 supplemented DMEM and TYB. Briefly, fresh rabbit brain tissue was incubated and the malondialdehyde (MDA) (forming because of auto oxidation) levels were calculated by adding thiobarbituric acid. MDA concentration of the final medium is calculated by its absorbance under a $532 \mathrm{~nm}$ spectrophotometer. Further calculations were done to convert the antioxidant capacity to a value given in percentage (11).

\section{Osmolarity Determination}

The osmolarity of DMEM, EGb761 supplemented DMEM and TYB/DMEM (1/1) were determined by an osmometer (Advanced Instruments Model 3300, USA). 


\section{Statistics}

Statistical analysis was based on independent sample t-tests for comparing means and values, where $p<0.05$ were considered to be significant.

\section{Results}

The harvested and pooled cornea epithelial cell number from 18 rabbits is $52 \pm 4 \times 10^{6}$ (mean \pm SD). The viability course of epithelial cells in two groups is mentioned in Fig. 2.

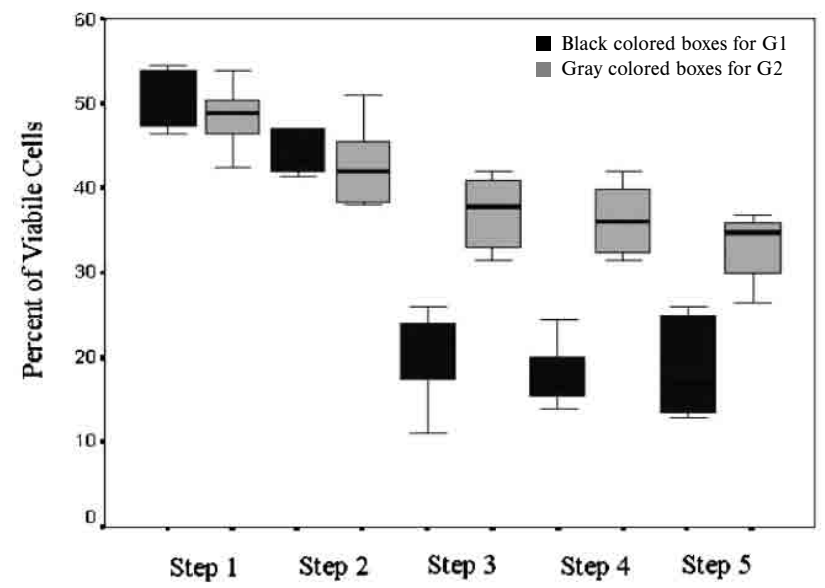

Fig. 2: The viability percent of corneal epithelial cells at each step.

Counts are repeated for six times for each step (n: 6).

Step 1: Before pretreatment incubation.

Step 2: 110 minutes after pretreatment incubation (with or without EGb 761) just before freezing.

Step 3: Immediately after thawing (time 0).

Step 4: 1 hour after thawing,

Step 5: 2 hours after thawing.

Before freezing there is no difference between groups, while after thawing comparison of means is significantly meaningful.

Viability values for step 1 and 2 were not different, while values for steps 3,4 and 5 were significantly different for G1 and G2 ( $\mathrm{p}<0,05)$. EGb 761 significantly improved viability values after thawing when compared with the control group. When treatment was done with EGb 761 before freezing, cell viability was improved almost twofold. Unfortunately, we couldn't find any reports at medical search databases to compare our freeze-thaw results with any other researchers.
The antioxidant capacity of the three mediums was determined and found to be $0 \%$ for DMEM, $76 \%$ for EGb $761(10.7 \% \mathrm{v} / \mathrm{v})$ supplemented DMEM and $32 \%$ for TYB/ DMEM $(1 / 1 ; \mathrm{v} / \mathrm{v})$. The osmolality of these mediums were respectively, 320, 870 and $1160 \mathrm{mOsm}$.

\section{Discussion}

Cryopreservation of whole cornea tissue is under the scope of current science (12) because of its potential for clinical applications (4). Our results may help for at least one cornea cell type (epithelia) during a freeze-thaw situation. Also, clinically, cornea freezing may occur during unprotected exposure to cold temperatures and severe winds during unprotected expouse in arctic region locations. In this situation the first layer to freeze. With the help of proper cryopreservation procedures we can perform further research for cornea epithelial cell biology, culture properties, and secretion of various autocrine and paracrine growth factors.

Freezing and thawing procedures cause chemical and physical stress to the cell membrane. Cell cryostorage technology strives to discover protective factors for the cell membrane, which is under stress of mechanical disruption caused by ice crystals and harmful effects of cryoprotectant agents during the freezing process. Crystallization of cytoplasm liquid components causes membrane rupture during freezing, while the osmotic pressure of cryoprotectants can dehydrate and be harmful to the cell, so a proper cooling speed/rate must be achieved. Also, there are reports that free radical levels may also play a role in freeze-thaw damage $(6,7)$. Also, these free radicals can potentially cause damage to the cell membrane during freeze-thaw procedures (13). Care must taken for a lot of parameters to achieve the optimal freeze-thaw application.

Our results show a beneficial effect of EGb 761 for cryostorage of rabbit corneal epithelial cell suspension. Although EGb 761 has a very wide spectrum of effects on biological tissues, the most well known and studied effect are their antioxidant effects. The success of EGb 761 extract in our study may be explained by these antioxidant properties. Based on our results one can postulate that adding an EGb 761 agent before freezing may also help the cells to recover from the destructive enzymatic and centrifugation based detrimental effects on at least membrane site. Other positive effects of EGb 761 may be due to the mechanism of protection against hypoxia (9) or protection of cell membrane components (3) shown on rat skeletal muscle and rat peripheral nervous cells, respectively.

Tab. 1: Viability percent mean values \pm standard deviations, $p$ value and mean of difference for each step.

\begin{tabular}{|l|c|c|c|c|}
\hline Step & 1 & 2 & 3 & 4 \\
\hline G1 viability (Blue) & $51,3 \pm 3,4$ & $45,2 \pm 5,1$ & $20,3 \pm 5,4$ & $17,8 \pm 3,8$ \\
\hline G2 viability (Orange) & $48,5 \pm 3,8$ & $42,8 \pm 5,0$ & $37,1 \pm 4,4$ & $36,3 \pm 4,2$ \\
\hline p value & 0,2 & 0,43 & 0,000 & 0,000 \\
\hline Mean of difference & 2,8 & 2,4 & $-16,8$ & $-18,5 \pm 5,7$ \\
\hline
\end{tabular}


To clarify the specific component of EGb761 responsible for the results obtained in this study is beyond our aim. Iinstead we accept our results as the effect of combined activity (aditive, antagonistic and synergistic) (5). One component in EGb 761 is that the flavonoids have protective effects on cell membranes against destructive effects of free radicals and lipid peroxidation (2). Another important group found in EGb 761, the terpenoids, exhibit significant antioxidant activity (8) and regulate some mitochondria enzyme properties (1). The number of components in EGb 761 is over 60. Therefore it is very hard to conclude that a specific component is responsible for the protective effects in the current study. It is likely several components acting together to provide the protection. One concern is that during filtering with $0.45 \mu \mathrm{m}$ pore nitrocellulose is perhaps a loss of extract components.

There is only one published paper on the use of EGb 761 while cooling entire rat bodies (10) for low temperature related studies. Our work is the first report using EGb 761 during the freezing-thawing of cells.

Direct transfer into the -80 degrees $\mathrm{C}$ freezer resulted in better post-cryopreservation growth in the culture.

Considering that all cell types and species may have unique properties, the results obtained in our study can be concluded for only New Zealand rabbit cornea cells. One must be aware that all other cells need their own specific evaluation. Our preliminary work was made on rat corneas and the results were in concordance with the current study. Controversially, deleterious results were obtained with hu- man spermatozoa freeze- thaw experimentation with EGb 761 pre-freezing treatment (unpublished data).

\section{References}

1. Amri H, Ogwuegbu SO, Boujrad N, Drieu K, Papadopoulos V. In vivo regulation of the peripheral-type benzodiazepine receptor and glucocorticoid synthesis by the Ginkgo Biloba extract EGb 761 and isolated ginkgolides. Endocrinology, 1996;137:5707-18

2. Artmann GA, Schikarski C. Gingko biloba extract (EGb 761) protects red blood cells from oxidative damage. Clin. Hemorheol, 1993;13:529-39.

3. Bruno C, Cuppini R, Sartini S, Cecchini T, Ambrogini P, Bombardelli E. Regeneration of motor nerves in bilobilade-treated rats. Planta Medica, 1993;59:302-7.

4. Castro-Munozledo F, Ozorno-Zarate J, Naranjo-Tackman R, Kuri-Harcuch W. Frozen cultured sheets of epidermal keratinocytes in reepithelialization and repair of the cornea after photorefractive keratectomy. J Cataract Refract Surg. 2002 Sep;28(9):1671-80.

5. DeFeudis, VF, Ginkgo biloba extract (EGb 761) From Chemistry to the Clinic. Ullstein Medical,Wiesbaden, Germany, 1998;p 2-15.

6. Lasso JL, Noiles EE, Alvarez JG, Storey BT. Mechanism of superoxide dismutase loss from human sperm cells during cryopreservation. J Androl 1994 MayJun;15(3):255-65.

7. Limaye LS. Bone marrow cryopreservation: improved recovery due to bioantioxidant additives in the freezing solution. Stem Cells 1997;15(5):353-8.

8. Pietri S, Maurelli E, Drieu K, Calcasi M. Cardioprotective and antioxidant effects of the terpenoid constituents of Ginkgo Biloba extract (EGb761). J. Mol. Cell. Cardiol, 1997;29:733-42.

9. Punkt K, Welt K, Schaffranietz L. Gingko biloba extract (EGb 761) protects enzyme activities in hypoxic rat myocardium. In: Natural Antioxidants: Molecular Mechanisms and Health Effects (L. Packer, M.G. Traber and W. Xin, eds), AOCS Press, Champaign, Illinois 1996; 466-71.

10. Ramachandran U, Divekar HM, Grover SK, Srivasta KK. New experimental model for the evaluation of adaptogenic products. J Ethnopharmacol 1990;29:275-81

11. Rice-Evans $\mathrm{C}$ and Miller NJ, Total antioxidant status in plasma and body fluids. Methods in Enzymology, 1994;234:279-85.

12. Routledge C, Armitage WJ. Cryopreservation of cornea: a low cooling rate improves functional survival of endothelium after freezing and thawing. Cryobiology. 2003 Jun;46(3):277-83.

13. Thomas MC, Anon MC. Study of the influence of freezing rate on lipid oxidation in fish (salmon) and chicken breast muscles. Int J Food Sci Technol 1990;25:718-21.

Submitted August 2006 Accepted October 2006.

\section{Corresponding author:}

Ass. Prof. Dr. Murad Aktan, Selcuk University, Meram Medical School, Department of Histology and Embriyology, 42080, Akyokus, Konya, Turkey, e-mail: muradaktan@gmail.com 